\title{
Energy Confinement Time and Heat Transport in Initial Neutral Beam Heated Plasmas on the Large Helical Device
}

H. Yamada, K. Y. Watanabe, S. Sakakibara, S. Murakami, M. Osakabe, O. Kaneko, K. Narihara, K. Tanaka, K. Ida, T. Minami, M. Goto, H. Idei, S. Inagaki, S. Kado, K. Kawahata, A. Komori, S. Kubo, J. Miyazawa, T. Morisaki, S. Morita, H. Nakanishi, S. Ohdachi, N. Ohyabu, Y. Oka, B. J. Peterson, R. Sakamoto, M. Shoji, H. Suzuki, Y. Takeiri, K. Toi, T. Tokuzawa, K. Tsumori, I. Yamada, K. Ohkubo, S. Sudo, K. Yamazaki, O. Motojima, M. Fujiwara, and LHD Experimental Group

National Institute for Fusion Science, Toki, Gifu 509-5292, Japan

(Received 2 August 1999)

\begin{abstract}
The confinement characteristics of large net-current-free plasmas heated by neutral-beam injection have been investigated in the Large Helical Device (LHD). A systematic enhancement in energyconfinement times from the scaling derived from the medium-sized heliotron/torsatron experiments have been observed, which is attributed to the edge pedestal. The core confinement is scaled with the Bohm term divided by the square root of the gyro radii. The comparative analysis using a dimensionally similar discharge in the Compact Helical System indicates gyro-Bohm dependence in the core and transport improvement in the edge region of LHD plasmas.
\end{abstract}

PACS numbers: $52.55 . \mathrm{Hc}, 52.25 . \mathrm{Fi}, 52.50 . \mathrm{Gj}$

The Large Helical Device (LHD) [1] has extended the envelope of a confinement study on currentless helical plasmas by more than 1 order of magnitude in plasma volume. LHD is a large superconducting heliotron with a major radius $R$ of $3.9 \mathrm{~m}$ and a minor radius $a$ of $0.6 \mathrm{~m}$. The experimental data of the medium-sized helical devices $(a=0.18-0.27 \mathrm{~m})$ have been accumulated in the international stallarator database, which has yielded the international stallarator scaling 95 (ISS95) [2];

$$
\tau_{E}^{\mathrm{ISS} 95}=0.079 \times a^{2.21} R^{0.65} P_{\mathrm{abs}}^{-0.59} \bar{n}_{e}^{0.51} B^{0.83} \boldsymbol{t}_{2 / 3}^{0.4} .
$$

Here the units of an energy confinement time $\tau_{E}$ in s, an absorbed heating power $P_{\text {abs }}$ in MW, and a line averaged electron density $\bar{n}_{e}$ in $10^{19} \mathrm{~m}^{-3}$ are used and $t_{2 / 3}$ is the rotational transform at the two-thirds radius (0.58 in LHD). This scaling satisfies the Kadomtsev constraint $[3,4]$ and is rewritten into the following dimensionless expression [5]:

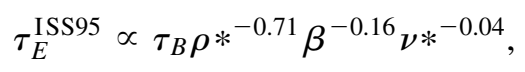

where $\tau_{B}, \rho *, \nu *$, and $\beta$ are, respectively, the Bohm diffusion time, the Larmor radius normalized by the plasma minor radius, collisionality, and the plasma kinetic pressure normalized by the magnetic field pressure. This expression indicates that confinement in helical systems is nearly gyro-Bohm. Since dependence on $\rho *$ has a great impact on extrapolation to reactor-grade plasmas, careful examination by a wide-range scan of $\rho *$ is prerequisite for establishment of a reliable scaling law. $\rho *$ is also connected to the characteristic scale length of a predominant instability; therefore, it is attracting interest in the clarification of the physical mechanism of anomalous transport. Exploration in LHD combined with past studies in medium-sized helical devices is highlighted in this strategy.

Parameter regimes studied here cover magnetic field $B$ of $1.5-2.75 \mathrm{~T}$, line averaged density $\bar{n}_{e}$ of $(1.0-4.9) \times$ $10^{19} \mathrm{~m}^{-3}$, and heating power of neutral beam injection
(NBI) $P_{\text {abs }}$ of $0.75-3.2 \mathrm{MW}$. Hydrogen discharges in the standard configuration which is characterized by the magnetic axis position $R_{\mathrm{ax}}$ of $3.75 \mathrm{~m}$ and the $n$ (toroidal mode number) $=0$ component of the ellipticity of 1 are studied. All correlation between sets of the logarithm of $\bar{n}_{e}, P_{\mathrm{abs}}$, and $B$ are less than 0.25 , which indicates that independence of surveyed parameters is sufficient. Diamagnetic measurement and a variety of profile measurements have been employed in this study. Port-through power of NBI is calibrated by the direct heat load measurement in case of no plasma target. Absorbed power, which is the sum of powers to electrons and ions, is then evaluated considering orbit and charge-exchange losses with the three dimensional Monte Carlo simulation [6]. The contribution of beam stored energy is included in the diamagnetic measurement; however, it is as low as $7 \%$ even in the lowest density case of $1 \times 10^{19} \mathrm{~m}^{-3}$.

Comparison of LHD data with available scaling laws, i.e., ISS95, the Lackner-Gottardi (LG) [7] scaling, the LHD scaling [8], and the gyro-reduced Bohm (GRB) scaling [9], indicates systematic enhancement of confinement. Enhancement factors are $1.18 \pm 0.15$ for ISS95, $1.15 \pm$ 0.13 for LG, $1.44 \pm 0.16$ for LHD, and $1.40 \pm 0.18$ for GRB. A regression analysis of LHD data alone gives

$$
\tau_{E}^{\mathrm{fit}}=0.05 \times P_{\mathrm{abs}}^{-0.57} \bar{n}_{e}^{0.61} B^{0.71},
$$

which is rewritten into

$$
\tau_{E}^{\mathrm{fit}} \propto \tau_{B} \rho *^{-0.83} \beta^{0.08} \nu *^{0.01} .
$$

The dependences on the key operational parameters, i.e., $P_{\mathrm{abs}}, \bar{n}_{e}, B$, are similar to ISS95. In particular, positive density dependence leads to the gyro-Bohm nature. Figure 1 shows the comparison of experimental data with the analyzed expression.

The database of LHD has been combined with the international stellarator database [2] and the medium-sized 


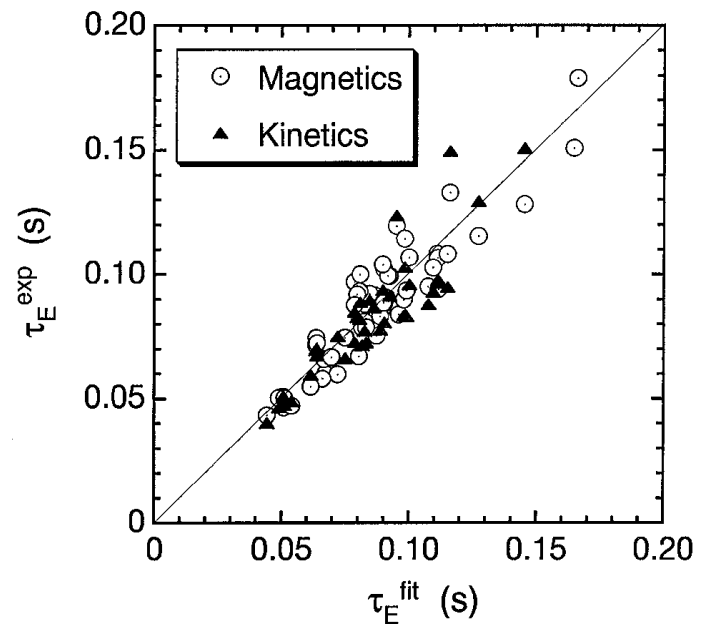

FIG. 1. Comparison of the energy confinement time obtained in the experiments versus the prediction from the fitted expression derived from the statistical analysis of LHD data alone.

heliotron/torsatrons, i.e., Heliotron-E, Advanced Test Facility (ATF), and Compact Helical System (CHS), which have a common magnetic configuration to LHD to explore the size effect on confinement, are selected. The regression analysis of the medium heliotron/torsatrons alone yields the scaling [2]

$$
\begin{aligned}
\tau_{E}^{\mathrm{scl} 1} & =0.04 \times a^{2.06} R^{0.74} P_{\mathrm{abs}}^{-0.63} \bar{n}_{e}^{0.53} B^{0.80} \boldsymbol{t}_{2 / 3}^{0.39} \\
& \propto \tau_{B} \rho *^{-0.50} \beta^{-0.33} \nu *^{0.06}(R / a)^{0.30} \boldsymbol{t}_{2 / 3}^{1.09} a^{-0.10} .
\end{aligned}
$$

The present data of LHD lies systematically well above this scaling [see Fig. 2(a)]. A simple statistical analysis of the combined database including LHD data gives an unusual expression:

$$
\tau_{E}^{\mathrm{a} l 1}=0.37 \times a^{3.22} R^{0.20} P_{\mathrm{abs}}^{-0.61} \bar{n}_{e}^{0.52} B^{0.86} \boldsymbol{t}_{2 / 3}^{0.93} .
$$

Although the dependences on $P_{\mathrm{abs}}, \bar{n}_{e}$ and $B$ are robust, the dimensional parameter $a$ cannot be eliminated in rewriting into a dimensionless expression

$$
\tau_{E}^{\mathrm{a} 11} \propto \tau_{B} \rho *^{-0.79} \beta^{-0.20} \nu *^{-0.02}(R / a)^{-1.04} \boldsymbol{t}_{2 / 3}^{2.36} a^{1.31} .
$$

This cannot be judged to be physically meaningful and calls into question what causes systematic confinement improvement in LHD.

The major characteristic of LHD plasmas which is different from the past medium heliotrons is the formation of an edge pedestal [10]. This is in contrast to the fact that the plasma pressure in medium-sized heliotrons decreases asymptotically to zero towards the last closed flux surface and suggests that confinement characteristics in LHD are different in the edge and in the core (see Fig. 3). In medium heliotrons, the part of the pedestal is considered to be much smaller. The decomposition of confinement region into the core and the pedestal has helped in
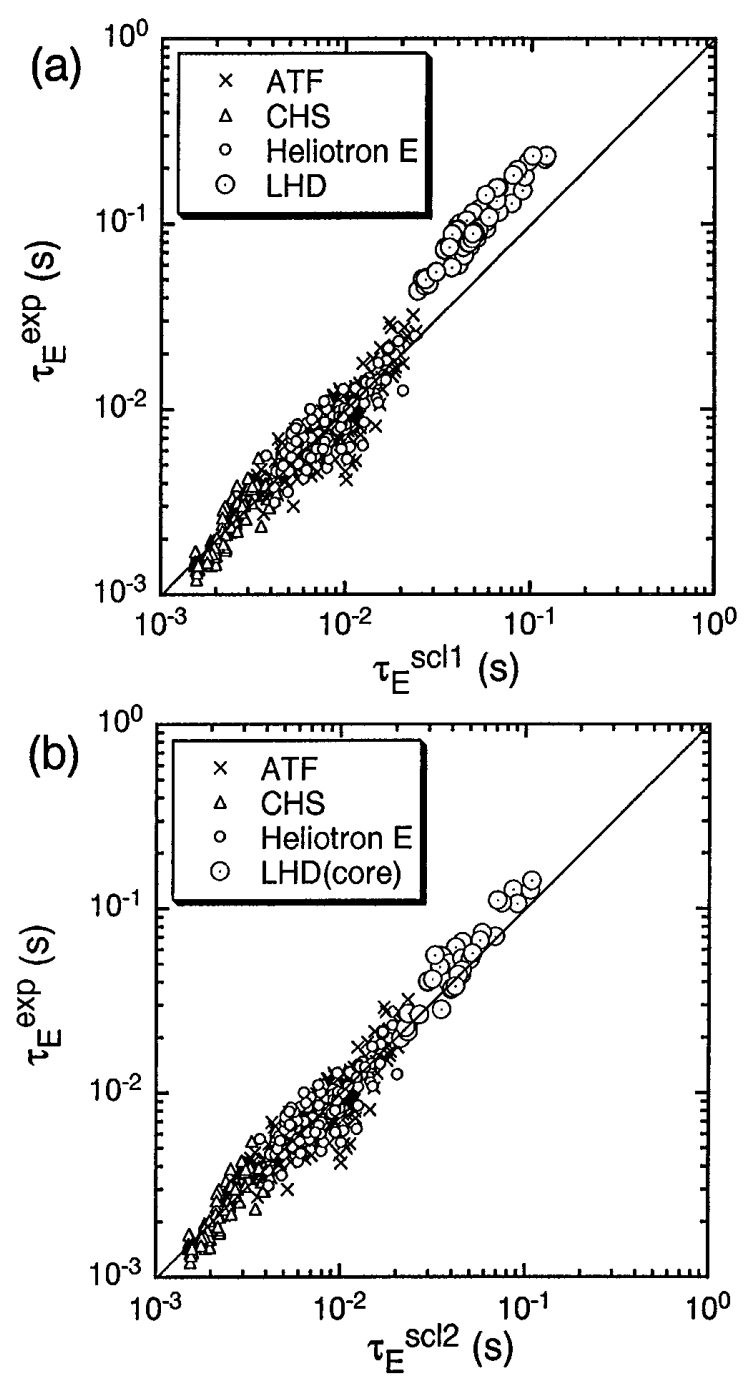

FIG. 2. Comparisons of the experimental energy confinement times versus the scalings. (a) The scaling derived from the precedent medium-sized experiments: Heliotron-E, ATF, and CHS. (b) The scaling derived from the combined data of the precedent medium-sized experiments and the contribution of the core $(\rho \leq 0.9)$ in LHD.

the understanding of characteristics of H-mode discharge in tokamaks [11]. The position of the "knee" is set at $\rho=0.9$, where $\rho$ is the normalized minor radius, for simplicity which generally agrees with the experimental observation. The core stored energy $W_{\text {core }}$ is defined by the subtraction of the pedestal component from the whole energy in the following integration of plasma pressure $p$ :

$$
W_{\text {core }}=\int_{\rho=0}^{\rho=0.9}[p(\rho)-p(0.9)] d v .
$$

The core stored energy by this definition in CHS is $91.2 \%$ of the total stored energy in average. Since all the data of medium-sized heliotron/torsatrons cannot be renewed, this concentration is assumed to all other data than in LHD to keep an equal footing as much as possible. A statistical 


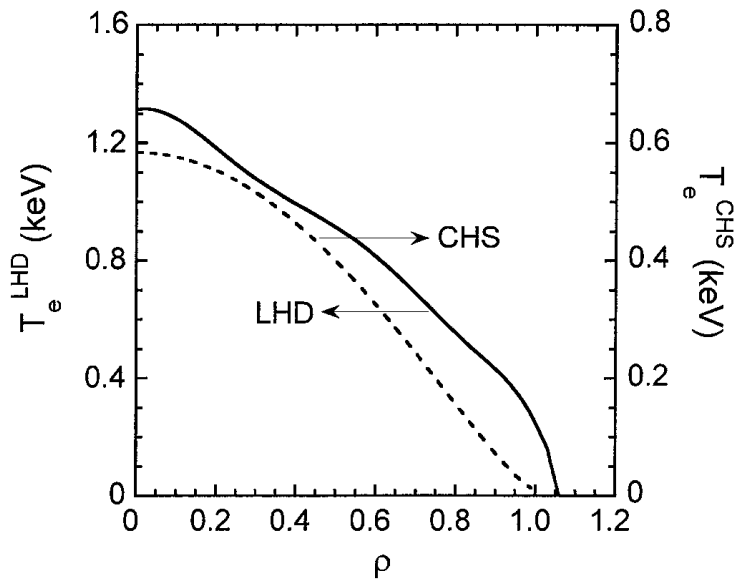

FIG. 3. Electron temperature profiles in LHD and CHS which are used in 1D transport analysis shown in Fig. 5. The magnetic fields are $1.5 \mathrm{~T}$ and $1.76 \mathrm{~T}$ for LHD and CHS, respectively.

analysis of data from the combination of the core confinement in LHD and the medium heliotrons gives the best fitted expression of

$$
\tau_{E}^{\mathrm{sc} 12}=0.059 \times a^{2.24} R^{0.67} P_{\mathrm{abs}}^{-0.63} \bar{n}_{e}^{0.54} B^{0.81} \boldsymbol{\epsilon}_{2 / 3}^{0.47} .
$$

Here the minor radius of the core is set as $0.9 a$. Figure 2(b) illustrates the comparison of experimental data of this scaling. This expression is close to that derived from the medium devices only [Eq. (1)] and rewritten into the following nearly dimensionally correct equation:

$$
\tau_{E}^{\mathrm{sc} 12} \propto \tau_{B} \rho *^{-0.53} \beta^{-0.33} \nu *^{0.07}(R / a)^{0.1} \boldsymbol{t}_{2 / 3}^{1.36} a^{0.05} .
$$

Confinement in the core region of LHD is nearly gyroBohm, which is consistent with the precedent understanding. A systematic enhancement of confinement can be attributed to the contribution from the pedestal.

All scalings for helical systems have indicated a positive density dependence unlike tokamak $L$-mode. The power density is supposed to be correlated with this saturation in tokamaks [2]. Figure 4 shows the density scan with the same heating power (1.5 MW). In the presently available range of density, confinement time can be scaled by $\bar{n}_{e}^{0.5-0.6}$. From the tokamak experience [5], saturation should occur at $\bar{n}_{e}=2 \times 10^{19} \mathrm{~m}^{-3}$. However, the positive density dependence is maintained beyond this predicted critical density. Since the positive density dependence of thermal energy has been pointed out in tokamak $L$-mode in JT-60 [12], further investigation with wider ranges in $P_{\mathrm{abs}}$ and $\bar{n}_{e}$ is required to clarify this issue.

A 1D local heat transport analysis also has been progressing to clarify confinement characteristics complementing global confinement analysis. $\nu *$ is important through neoclassical transport in the collisionless regime in helical plasmas when anomalous transport is suppressed sufficiently. Since $\rho *$ cannot be scanned over a wide

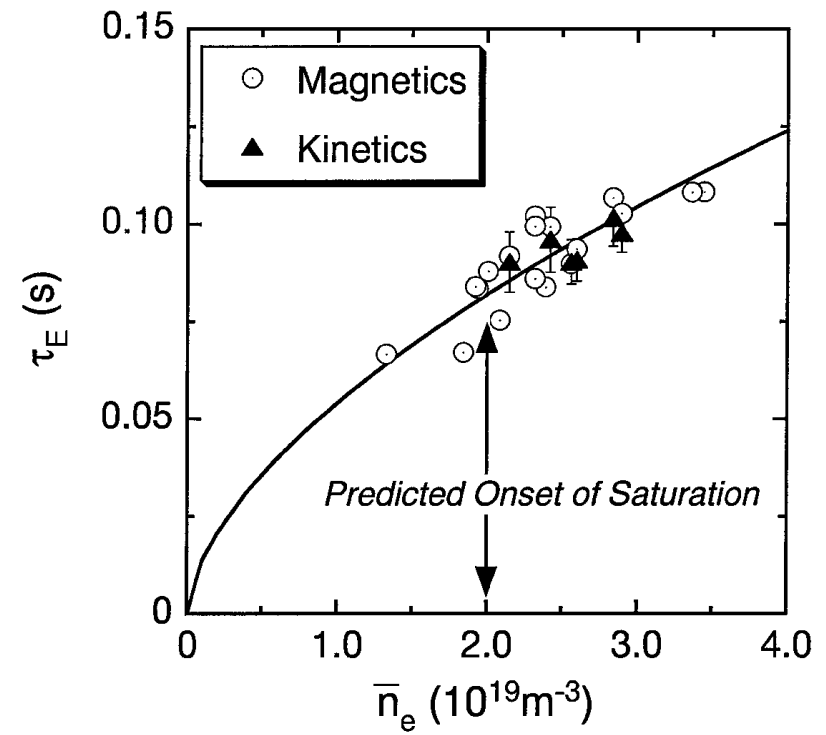

FIG. 4. Dependence of energy confinement times on density with the fixed heating power. A solid line expresses the dependence of $\bar{n}_{e}^{0.6}$ as reference.

range in a single device, comparison of experiments in two devices with different sizes is effective. Figure 5(a) shows the radial profiles of $\nu^{*}, \rho *$, and $\beta$ in the hot-ion mode discharge in CHS $(a=0.20 \mathrm{~m})$ and a typical discharge in LHD shown in Fig. 3. Although transport of NBI heated plasmas in CHS is generally dominated by anomalous transport, ion confinement in the core region $(\rho<0.7)$ is improved and close to the neoclassical prediction in the hot-ion mode [13]. Here two discharges with similar $\nu *$ are chosen. A normalized gyroradius of CHS is larger than that of LHD by a factor of 2 in $\rho<0.7$. Since the high edge temperature distinguishes LHD from CHS, their collisionality and normalized gyroradius show different trends in the peripheral region. Since the temperature drops towards zero as the edge is approached in CHS, the collisionality increases rapidly and the gyroradius decreases. In LHD both change much more gradually. The physical picture of gyro-Bohm-type transport predicts that heat transport is improved in LHD due to smaller $\rho *$ in $\rho<0.9$. However, confinement in LHD is expected to be degraded in the edge region compared with CHS because of larger $\rho *$. Since the Bohm factor $\left(\chi_{B} \propto T / B\right)$ of LHD is twice as large as that of CHS, heat conduction should be the same in the core region. Figure 5(b) shows the electron heat conduction coefficients $\chi_{e}$ of CHS and LHD. In the core region, LHD is better than CHS by a factor of 2-3. The heat conduction in CHS is much enhanced in the edge region while LHD has an almost flat profile. With regard to comparison with neoclassical theory, a deterioration factor is suppressed up to around 3 in LHD although it has the order of 10-100 in CHS. However, anomalous transport is still dominant in electron heat transport in LHD although collisionality enters the collisionless regime where helical ripple transport plays 

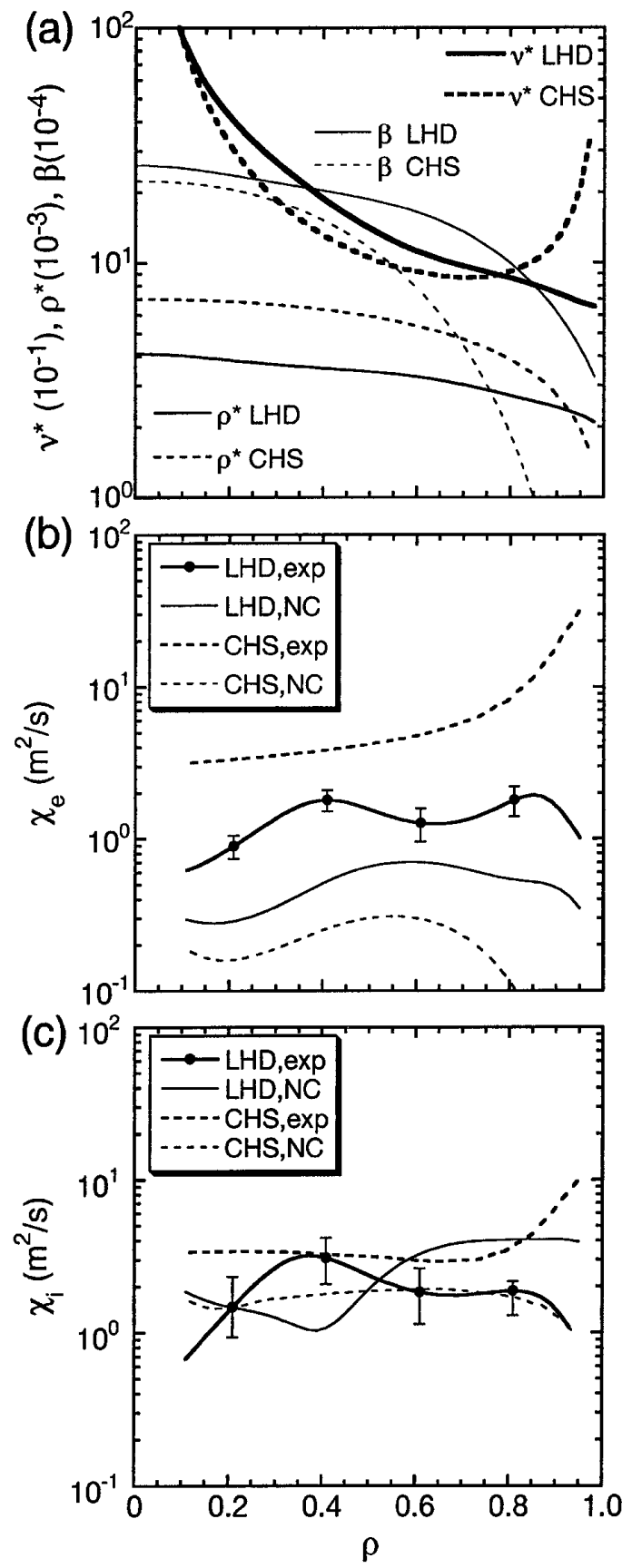

FIG. 5. Comparison of two dimensionally similar discharges in CHS and LHD. (a) Radial profiles of $\rho *, \nu *$, and $\beta$. (b) Radial profile of the electron heat conductivity $\chi_{e}$. The suffix, NC, denotes the prediction from a neoclassical theory. (c) Radial profile of the ion heat conductivity $\chi_{i}$. Typical error bars are illustrated every one-fifth radius.

an essential role. Differences in the ion transport of CHS and LHD is smaller than in electrons except for the edge region [see Fig. 5(c)]. Deterioration in the edge region is observed in CHS as in the electron transport. In contrast to electrons, ion transport is close to neoclassical in both CHS and LHD. The radial electric field effect is not included here in LHD since the experimental result is not available to date while it is included for CHS [13]. If the comparable potential to the temperature is assumed in LHD, the heat conduction is suppressed by $50 \%$ and $15 \%$ for ions and electrons, respectively. The difference in heat transport coefficients between CHS and LHD supports the physical picture of gyro-Bohm in the core region. The values of both heat conduction coefficients for ions and electrons are close to each other in LHD. This fact also suggests that the predominant instability is electrostatic, which is consistent with gyro-Bohm-type transport. If $\rho *$ dependence does not exist, the transport in LHD should be worse by a factor of 2 than in CHS. In the edge region, however, experimental observation is clearly inconsistent with gyro-Bohm-type transport. The suppression of heat transport in the edge region of LHD plays an essential role in the formation of the pedestal which is not observed in CHS. This is also phenomenologically consistent with the experimental observation that confinement deteriorates when the edge pedestal is lost due to a strong gas puff or strong magnetic perturbation by external coils inducing a large island at the edge. Although the $\rho *$ dependence of LHD plasmas $\left(\rho *^{-0.83}\right)$ is stronger than Eq. (2) $\left(\rho *^{-0.53}\right)$, it is weakened when only the core part is considered.

The authors thank A. Iiyoshi for his continuous encouragement throughout this study. The authors gratefully acknowledge the operational support of all members of the device engineering group.

[1] O. Motojima et al., Phys. Plasmas 6, 1843 (1999).

[2] U. Stroth et al., Nucl. Fusion 36, 1063 (1996).

[3] B. B. Kadomtsev, Sov. Phys. J. Plasma Phys. 1, 295 (1975).

[4] J. W. Connar and J. B. Taylor, Nucl. Fusion 17, 1047 (1977).

[5] U. Stroth, Plasma Phys. Controlled Fusion 40, 9 (1998).

[6] S. Murakami, N. Nakajima, and M. Okamoto, Trans. Fusion Technol. 27, 256 (1995).

[7] K. Lackner and N. A. O. Gottardi, Nucl. Fusion 30, 767 (1990).

[8] S. Sudo et al., Nucl. Fusion 30, 11 (1990).

[9] M. Murakami et al., Phys. Fluids B 3, 2261 (1991).

[10] N. Ohyabu et al., Phys. Rev. Lett. 84, 103 (2000).

[11] J. G. Cordey et al., Nucl. Fusion 39, 301 (1999).

[12] T. Takizuka, in Proceedings of the 1992 International Conference on Plasma Physics, Innsbruck, Austria, edited by W. Freysinger et al. (The European Physical Society, Geneva, 1992), Vol. 16C, Pt. I, p. 51.

[13] K. Ida et al., Nucl. Fusion (to be published). 